\title{
Preparation and Characterization of a Poly(strong base) with Narrow Molecular Weight Distribution; Poly(4-vinylbenzyltrimethylammonium chloride)
}

\author{
Yuji Higo, Haruhisa Chosi, Teruo Fujimoto, ${ }^{\dagger}$ \\ and Mitsuru NAGASAWA \\ Department of Synthetic Chemistry, Nagoya University, \\ Furo-cho Chikusa-ku, Nagoya 464, Japan.
}

(Received March 13, 1980)

\begin{abstract}
In order to obtain a cationic polyelectrolyte with a narrow molecular weight distribution, the anionic polymerization of 4-vinylbenzyldimethylamine was studied. It was concluded that, if the polymerization of the monomer is carried out in benzene with s-butyllithium at low monomer concentrations ( $\mathrm{ca} .3 \%$ ), the polymerization proceeds at a first-order reaction rate and the resulting poly(4-vinylbenzyldimethylamine)s each have narrow molecular weight distributions. Moreover, it was found that the living-ends in this polymerization system are ionpairs, and are not associated in the benzene solution. Poly(4-vinylbenzyldimethylamine) can be converted to a salt of the strong base, poly(4-vinylbenzyltrimethylammonium iodide) by quaternization with methyl iodide. No chain degradation is observed.
\end{abstract}

KEY WORDS Anionic Polymerization / 4-Vinylbenzyldimethylamine / Molecular Weight Distribution / Cationic Polyelectrolyte / Poly(4-vinylbenzyltrimethylammonium chloride) /

It is needless to stress the importance of the samples having sharp molecular weight distributions in order to study the physical properties of polymers. This applies to study of polyelectrolytes as well. We now have poly(strong acid) ${ }^{1}$ and poly(weak acid) ${ }^{2}$ having sharp molecular weight distributions. The purpose of this work is to study the method of preparing poly(4-vinylbenzyldimethylamine) (P4VBDMA) which has a narrow molecular weight distribution. P4VBDMA can easily be converted to a salt of strong base, i.e., poly(4-vinylbenzyltrimethylammonium salt).

\section{EXPERIMENTAL}

\section{Preparation of 4-Vinylbenzyldimethylamine}

$\beta$-Phenylethyl bromide was chloromethylated with chloromethyl methyl ether in carbon tetrachloride in the presence of zinc chloride. The reaction

$\dagger$ Present address: Department of Material Science and Technology, Technological University of Nagaoka, Kamitomioka-cho, Nagaoka 949-54, Japan. product was a mixture of ortho and para isomers. The $\beta$-(4-chloromethylphenyl)ethyl bromide was separared from the reaction mixture by recrystallization in a mixed solvent of hexane and benzene. The $\beta$-(4-chloromethylphenyl)ethyl bromide was converted to $p$-chloromethylstyrene with potassium $t$-butoxide in dry $t$-butyl alcohol and made to react with dimethylamine in ethyl acetate at $237 \mathrm{~K}$.

The 4-vinylbenzyldimethylamine (4VBDMA) thus obtained was twice purified by extraction from ethyl acetate solutions containing ether with aqueous solution of $\mathrm{HCl}$. After the aqueous solution was neutralized with $\mathrm{NaOH}$, 4VBDMA was extracted with ether and was fractionally distilled: bp 366$370 \mathrm{~K}$ [2 $\mathrm{mmHg}(0.27 \mathrm{~Pa})]$.

The molecular structure of 4VBDMA thus obtained was confirmed by Varian 100 NMR spectrometer. After drying with calcium hydride, the 4VBDMA was further purified in the presence of benzophenone sodium. Distillation was repeated until the color of the anion remained unchanged during distillation. The monomer thus obtained was kept in a sealed flask at $253 \mathrm{~K}$. 


\section{Initiator}

$s$-Butyllithium ( $s$-BuLi) was prepared by the method of Smith et al. ${ }^{3}$ The concentration of the initiator was determined by titration with a standard $\mathrm{HCl}$ solution.

\section{Solvent}

The benzene used for polymerization was distilled with addition of butyllithium after the usual purification. The toluene, cyclohexane, and heptane used for characterization of the polymer was fractionally distilled after being washed with sulfuric acid and dried with sodium metal wire. The decane and heptane used for fractionation were of GR grade, from Tokyo-Kasei Co.

\section{Polymerization}

The rate of polymerization was determined by dilatometry. The apparatus and technique in this experiment are similar to that reported by Kitano et $a l .{ }^{4}$ The inner diameter of each capillary $(0.5-1.0$ $\mathrm{mm}$ ) was determined with mercury. An ampule enclosing the initiator solution together with a magnetic stirrer tip was enclosed in a glass cell containing the monomer solution. The initiator ampule was broken by turning the magnetic stirrer, so that the initiator could be mixed instantly with the monomer. The conditions for polymerization are shown in each figure caption.

The starting time of polymerization could be easily observed by the change in color of the solution. After the polymerization was completed, the dilatometer was immersed in liquid nitrogen and was opened. To kill the living-ends, methyl alcohol was added to the sample solution. The polymer solution was first freeze-dried, washed with water to remove the initiator, washed with cold hexane $(195 \mathrm{~K})$ and then dried in vacuo to determine the polymer yield.

The concentration of the living-ends [LE] could be calculated from the polymer yield and the number-average molecular weight of the polymer.

The samples for characterization were obtained by polymerization in a considerable yield as reported by Kitano et al. ${ }^{5}$ In order to measure the molecular weight and its distribution, the samples obtained were carefully purified by precipitation from a hexane solution by lowering the temperature $(195 \mathrm{~K})$. The polymerization conditions for larger yields are listed in Table I.
Preparation of Poly(4-vinylbenzyltrimethylammonium chloride)

P4VBDMA thus prepared was dissolved in a small amount of dry methyl alcohol, and diluted with dimethylformamide up to about $1 \%$. Dry methyl iodide was added to the solution and the reaction was continued with stirring for 24 hours at room temperature. The solutions was protected from light by covering the apparatus with aluminum foil. The reaction product was precipitated and washed with ethyl acetate and dried in vacuo. The degree of quaternization was checked by the potentiometric titration method. That is, poly(4-vinylbenzyltrimethylammonium iodide) (P4VBTMA-I) was dissolved in water and the solution was passed through a mixed bed column of Amberlite IR-120B and IRA-400. The base solution was titrated with a standard $\mathrm{HCl}$ solution using a Beckman Research $\mathrm{pH}$ meter. The degree of quaternization was calculated from the titration curve and the quantity of the sample used. The samples were stored in the chloride form.

\section{Molecular Characterization}

The number-average molecular weight $M_{n}$ was determined by osmometry in toluene at $298 \mathrm{~K}$ or $303 \mathrm{~K}$ with a high-speed membrane osmometer type 502 of Hewlet Packard Co. The weight-average molecular weight $M_{w}$ was determined by a FICA 4200 light scattering photometer with $546 \mathrm{~nm}$ neutral light in cyclohexane at $298 \mathrm{~K}$ and in heptane at $312 \mathrm{~K}$. The refractive index increments in the same solvent were determined by a Woods differential refractometer and were found to be $0.127( \pm 0.003)$ and $0.201( \pm 0.001) \mathrm{cm}^{3} \mathrm{~g}^{-1}$, respectively. The number-average molecular weight of P4VBTMA-Cl obtained by quaternization of P4VBDMA was determined by osmometry in a $0.5 \mathrm{~N} \mathrm{NaCl}$ solution at $303 \mathrm{~K}$.

The P4VBDMA sample could be fractionated with respect to molecular weight from a heptane or a decane solution by changing the temperature. The $\theta$-temperature in heptane is about $312 \mathrm{~K}$. Sample TH-3 was fractionated from an decane solution within the range of $273-292 \mathrm{~K}$. The integral molecular weight distribution curves were obtained by the method of Schulz. ${ }^{6}$ The molecular weight dispersity index, $M_{w} / M_{n}$, was calculated from the integral molecular weight distribution curves. 


\section{RESULTS AND DISCUSSION}

\section{Polymerization}

In polymerization in a dilatometer, the difference between the meniscus height at time $t(h)$ and that at time zero $\left(h_{0}\right)$ is proportional to the extent of conversion $x$. If the meniscus height at complete conversion is expressed by $h_{1}$, the value of $x$ at time $t$ is given by

$$
1-x=\left(h-h_{1}\right) /\left(h_{0}-h_{1}\right)
$$

If the rate of initiation is much faster than that of propagation, the polymerization rate can be ex-
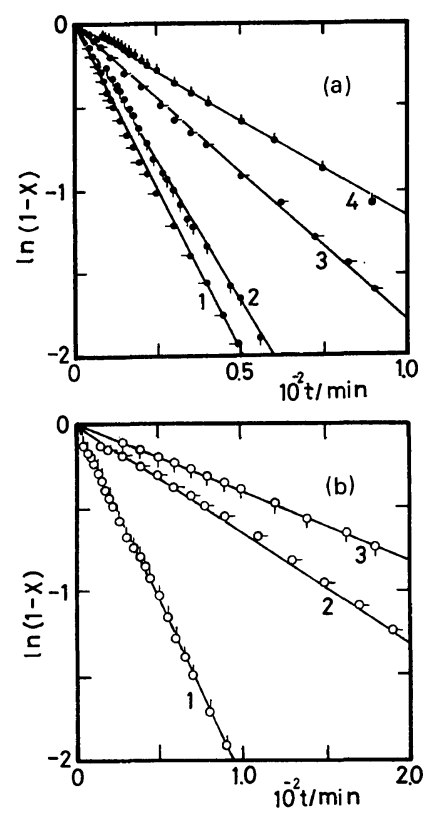

Figure 1. The first-order rate plots for the polymerization of 4VBDMA with $s$-BuLi.

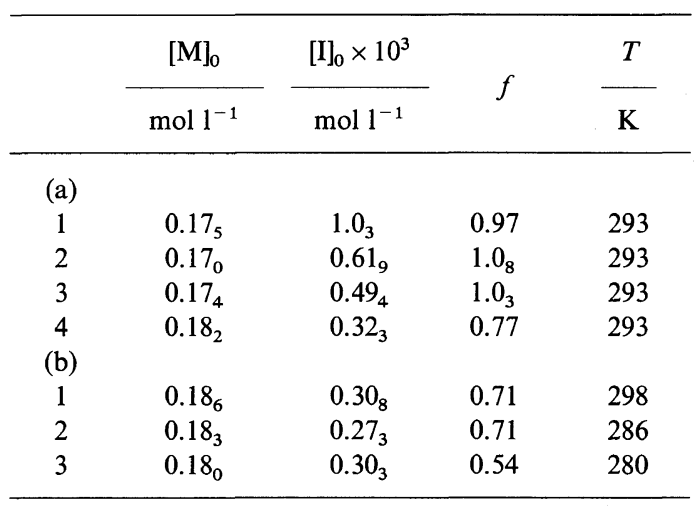

pressed by

$$
\mathrm{d}(1-x) /\left(\mathrm{d} t=-k_{\mathrm{ap}}(1-x)\right.
$$

where $k_{\text {ap }}$ is the apparent rate constant and is a function of the concentration of living-end. If eq 2 is integrated, we have

$$
\ln \left(h-h_{1}\right)=-k_{\mathrm{ap}} t+\ln \left(h_{0}-h_{1}\right)
$$

from which we can determine $k_{\text {ap }}$ and $\left(h_{0}-h_{1}\right)$.

If the solvent is a hydrocarbon, the living-ends are generally ion-pairs which associated with each other and the propagation is caused only by the unassociated ion-pair. Thus, $k_{\text {ap }}$ becomes

$$
k_{\mathrm{ap}}=k_{\mathrm{p}}[\mathrm{LE}]^{\alpha}
$$

where $k_{\mathrm{p}}$ is the propagation rate constant, [LE] is the toral concentration of the living-end and $\alpha$ gives the degree of association of a living-end. ${ }^{7}$

The polymerization kinetics in this system show different behaviors for different concentration ranges. It should be noted that the conversions attained $100 \%$ in all experiments. Typical examples of $\ln (1-x)$ vs. $t$ plots in benzene at lower concentrations of monomer ( $\mathrm{ca} .3 \%$ ) are shown in Figures 1a and $1 \mathrm{~b}$. In this case, a good linear relationship holds, independent by either initiator concentration or temperature.

$k_{\text {ap }}$ can be calculated from the slopes of the linear plots in Figures 1a and 1b. If plot $k_{\mathrm{ap}}$ against the living end concentration [LE] in a double logarithmic way, we have Figure 2, from which we find $\alpha=1$. That is, the propagation rate in benzene is of the first order with respect to the living end concentration $[\mathrm{LE}]$, meaning that there is no association of ion-pairs. ${ }^{7}$ The Arrhenius plots of the polymerization rate constants, i.e., the plots of $k_{\mathrm{p}}=k_{\mathrm{ap}} /[\mathrm{LE}] v s .1 / T$ are shown in Figure 3 . The

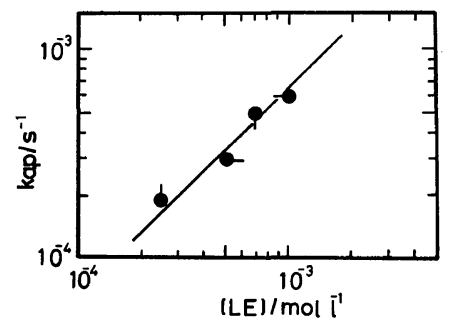

Figure 2. Plot of the apparent polymerization rate constant $K_{\text {ap }}$ against the living-end concentration in the experiments of Figure 1a. 


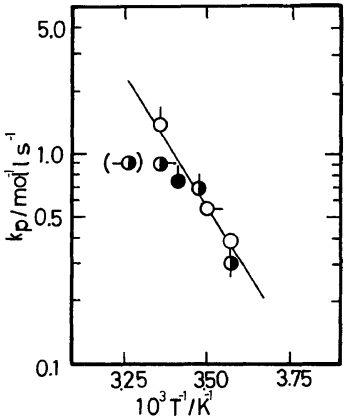

Figure 3. The Arrhenius plot of the propagation rate constant $k_{\mathrm{p}}$ against $T^{-1}$, for the experimental data in Figures 1 and 4. Symbols are the same as in Figures 1 and 4.

activation enthalpy of the propagation reaction is about $8 \mathrm{kcal} \mathrm{mol}^{-1}$, and this is in good agreement with the activation enthalpy of the propagation of styrene using the initiators of the ion-pair type in benzene, as reported by Bywater et al. ${ }^{7,8}$

The living-ends of an ion-pair type such as the styryl anion or isopropenyl anion generally associate with each other in benzene. The active end of P4VBDMA in benzene is also an ion-pair, but there is no association between the active ends. The reason for this phenomenon is perhaps that $\mathrm{Li}^{+}$ may be highly solvated with the amine groups of the monomer, thus preventing the association of ionpairs. ${ }^{9}$ If the polymerization is carried out at higher monomer concentrations ( $\mathrm{ca}$. 10\%), a linear relationship between $\ln (1-x)$ and $t$ does not hold for any initiator concentration or temperature, as can be seen in Figure 4. The first-order reaction rate is limited to a $30-50 \%$ conversion. If the rate constant is estimated at the initial stage in Figure 4, however, $k_{\mathrm{p}}$ fits the same line as those at low monomer concentrations in Figure 3. The reason for the deviation from the first-order reaction rate in Figure 4 is not clear. Our speculation is that the amine group of the polymer may participate in the solvation of $\mathrm{Li}^{+}$because of high affinity of amine group for $\mathrm{Li}^{+}$, thus having the effect of a polymerization mechanism. Consequently, if the concentration of the polymer is low, the anionic polymerization of 4VBDMA proceeds at a firstorder reaction rate with respect to the monomer concentration. However, if the concentration of polymer becomes high, the polymerization rate may deviate from the first-order reaction rate.

So, it may be considered that the polymer of

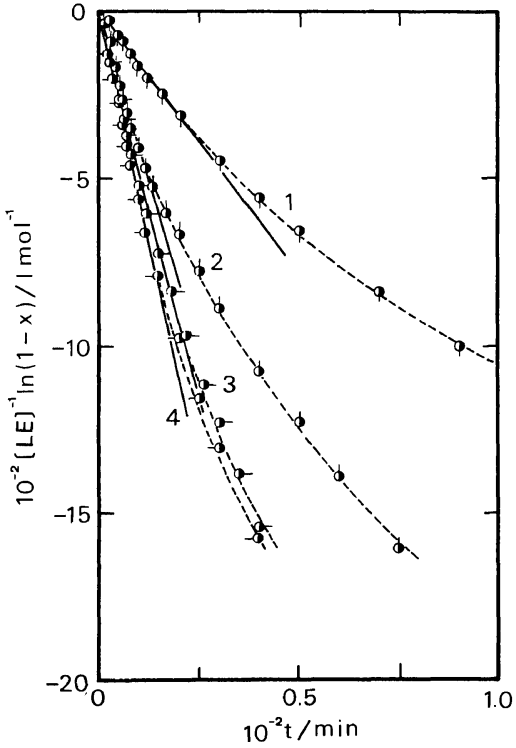

Figure 4. The first-order rate plots for the polymerization of 4VBDMA with $s$-BuLi.

\begin{tabular}{|c|c|c|c|c|}
\hline & {$[\mathrm{M}]_{0}$} & {$[\mathrm{I}]_{0} \times 10^{3}$} & & $T$ \\
\hline & $\mathrm{mol} \mathrm{l}^{-1}$ & $\mathrm{~mol} \mathrm{l}^{-1}$ & & $\mathrm{~K}$ \\
\hline 1 & $0.57_{1}$ & $1.6_{5}$ & 0.84 & 279.8 \\
\hline 2 & $0.56_{1}$ & $1.6_{7}$ & 0.77 & 288 \\
\hline 3 & $0.55_{2}$ & $1.5_{2}$ & 0.77 & 298 \\
\hline 4 & $0.57_{6}$ & $1.8_{7}$ & 0.83 & 306 \\
\hline
\end{tabular}

4VBDMA having a sharp molecular weight distribution, can be obtained provided the anionic polymerization of 4VBDMA with $s$-BuLi in benzene is carried out at monomer concentrations as low as $3 \%$.

\section{Molecular Weight Distribution of P4VBDMA}

About $5 \mathrm{~g}$ of monomers were polymerized with $s$ $\mathrm{BuLi}$ in benzene to examine the molecular weight distribution of the polymer. The experimental conditions are shown in Table I. The polymer molecular weight distributions determined by the fractionation method are shown in Figure 5. The molecular weight distribution index calculated from the molecular weight distribution curves are also listed in Table I.

It can be concluded from the data in Table I that sample TH-3 polymerized at about 3\% monomer concentration has a sharp molecular weight distri- 
Table I. Polymerization of 4VBDMA in a larger scale

\begin{tabular}{|c|c|c|c|c|c|c|c|c|}
\hline \multirow{2}{*}{ Sample } & {$[\mathrm{M}]_{0}$} & {$\left[\mathrm{I}_{0} \times 10^{3}\right.$} & $T$ & $\begin{array}{l}\text { Reaction } \\
\text { time }\end{array}$ & Yiled & \multirow{2}{*}{$M_{w} \times 10^{-5}$} & \multirow{2}{*}{$M_{n} \times 10^{-5}$} & \multirow{2}{*}{$M_{w} / M_{n}{ }^{2}$} \\
\hline & $\mathrm{mol} \mathrm{1}^{-1}$ & $\mathrm{~mol} \mathrm{1}^{-1}$ & K & $\mathrm{h}$ & $\%$ & & & \\
\hline TH-3 & $0.17_{9}\left(2.2_{8} \%\right)$ & $0.48_{1}$ & 288 & 24 & 100 & $1.26 \pm 0.05$ & $1.24 \pm 0.02$ & 1.06 \\
\hline TH-4 & $0.73_{3}(11.8 \%)$ & $1.7_{9}$ & 288 & 24 & 100 & $1.56 \pm 0.1$ & $1.43 \pm 0.02$ & 1.27 \\
\hline TH-7M & $0.14_{8}\left(2.3_{8} \%\right)$ & $0.62_{4}$ & 288 & 20 & 100 & - & - & - \\
\hline TH-8M & $0.16_{8}\left(2.7_{0} \%\right)$ & $0.29_{4}$ & 288 & 16 & 100 & - & - & - \\
\hline
\end{tabular}

${ }^{a}$ Caluculated from the molecular weight distribution data in Figure 5.

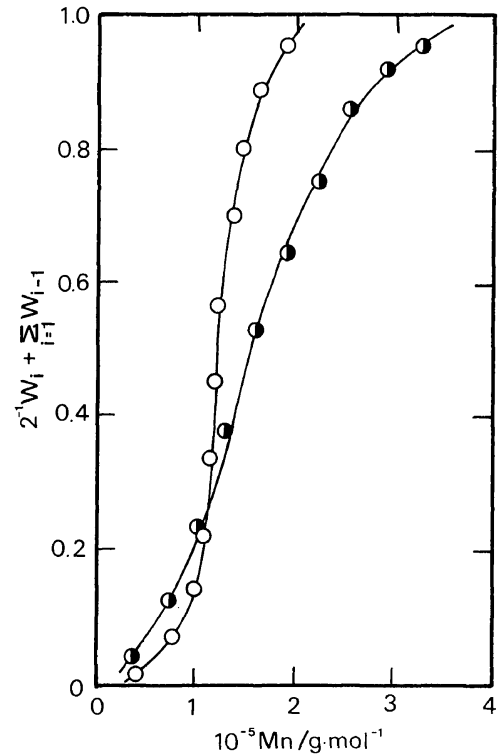

Figure 5. Molecular weight distributions of P4VBDMA: $\bigcirc$, sample TH-3; $\boldsymbol{O}$, sample TH-4.

bution, while the molecular weight distribution of sample TH-4 polymerized at a higher monomer concentration is clearly a broader case.

Finally, the anionic polymerization of 4VBDMA in THF was also studied. By analyzing the data by the method of Szwarc, ${ }^{7}$ it was found that the dissociation constant of an ion-pair may be very small. Probably for this reason, all samples obtained in THF have broad molecular weight distributions. We made no further investigation of the anionic polymerization of 4VBDMA in THF.

\section{Preparation of P4VBTMA-Cl}

Examples of potentiometric titration curves of the quaternized P4VBDMA are shown in Figure 6. The

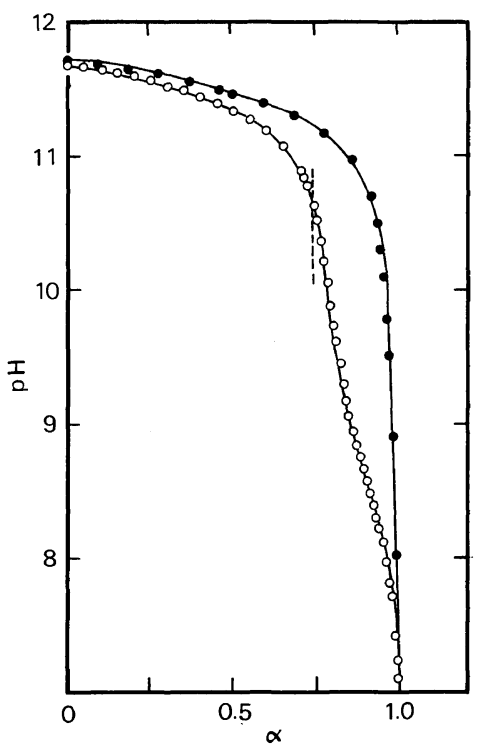

Figure 6. Potentiometric titration curves of P4VBTMA base: $O$, sample TH-7M-A; , sample TH-7M-B.

sample TH-7M-A was obtained from a mixture of P4VBDMA and methyl iodide in a mole ratio of $75: 25$, while sample TH-7M-B was obtained from the mixture having an excess amount of methyl iodide. The inflexion point in the potentiometric titration curve of sample TH-7M-A was found at $75: 25$, in agreement with the preparation data. Therefore, it is clear that the quaternization of the sample TH-7M-B is $100( \pm 1) \%$. The degree of polymerization determined before and after the quaternization reaction is shown in Table II. There was no degradation of polymer chain in the quaternization reaction with methyl iodide.

Finally, the concentration of living-ends [LE] in the polymerization solution was generally lower 
Table II. Number-average molecular weight before and after quarternarization ${ }^{\mathrm{a}}$

\begin{tabular}{cll}
\hline Sample & \multicolumn{1}{c}{ Before } & \multicolumn{1}{c}{ After } \\
\hline TH-7M & $5.4 \times 10^{4}(338)$ & $7.2 \times 10^{4}(339)$ \\
TH-8M & $1.66 \times 10^{5}(1030)$ & $2.13 \times 10^{5}(1010)$ \\
\hline
\end{tabular}

a The values in parenthesis are the degree of polymerization.

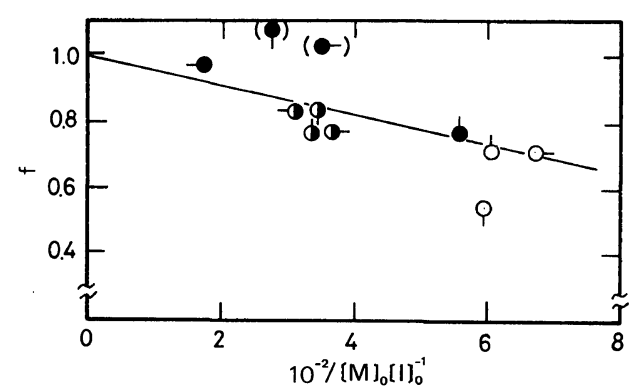

Figure 7. Plot of the initiation efficiency $f$ against $[\mathrm{M}]_{0} /[\mathrm{I}]_{0}$. Symbols are the same as in Figures 1 and 4 .

than the concentration of the initiator added to the system $[\mathrm{I}]_{0}$. That is, the initiation efficiency $f$, which is the ratio $[\mathrm{LE}] /[\mathrm{I}]_{0}$, is lower than unity. The values of $f$ are shown in the Figure captions. If we assume that the monomer still contains a certain amount of impurity which kills the initiator, the initiation efficiency $f$ should be linear to the ratio of the initial concentration of monomer $[\mathrm{M}]_{0}$ to the initial concentration of initiator $[\mathrm{I}]_{0}$. Thus,

$$
f=1-K[\mathrm{M}]_{0} /[\mathrm{I}]_{0}
$$

4 are in agreement with eq 5, as shown in Figure 7. Thus, it is certain that a certain amount of impurities are still present in the monomer. Consequently, it was difficult to obtain samples having higher molecular weights in the present work.

Acknowledgement. This work was initiated on the suggestion of Professor R. Asami of Nagoya Institute of Technology. We should like express our appreciation to Professor Asami for his suggestions and also to Dr. Y. Kawakami of Nagoya University for his helpful advice. The quaternization experiments were carried out with the cooperation of Mr. N. Kozuka, to whom our sincere thanks are also given.

\section{REFERENCES}

1. W. R. Carroll and H. Eisenberg, J. Polym. Sci., A-2, 4, 599 (1966).

2. T. Kitano, T. Fujimoto, and M. Nagasawa, Polym. J., 9, 153 (1977).

3. B. Smith and E. E. Turner, J. Chem. Soc., 861 (1953).

4. T. Kitano, M. Mitsumura, T. Fujimoto, and M. Nagasawa, Macromolecules, 8, 382 (1975).

5. T. Kitano, T. Fujimoto, and M. Nagasawa, Macromolecules, 7, 719 (1974).

6. G. V. Schulz, Z. Phys. Chem., B47, 155 (1940).

7. M. Szwarc, "Carbanions Living Polymers and Electron Transfer Process," Interscience, New York, N.Y., 1968.

8. D. J. Worsfold and S. Bywater, Can. J. Chem., 38, 189 (1960).

9. L. Reich and A. Schindler "Polymerization by Organometallic Compounds," Polymer Review, vol. 12, Interscience, New York, N.Y., 1966.

where $K$ is a constant. The data of $f$ in Figures 1 and 